\title{
FORMANDO JUVENTUDES: ESTADO DEL ARTE DE LAS PROPUESTAS FORMATIVAS EN EL ÁREA DE LA SALUD CON JÓVENES EN MEDELLIIN, COLOMBIA 2000-2006
}

\author{
FORMING YOUTHS: STATE OF THE ART OF FORMATIVE \\ PROPOSALS IN THE FIELD OF HEALTH WITH YOUTHS OF \\ MEDELLIN, COLOMBIA 2000-2006
}

\section{Nancy Eliana Gallo Restrepo*}

RESUMEN

\begin{abstract}
Esta investigación tiene el propósito de levantar un estado del arte de las propuestas formativas en el ámbito de la salud, orientadas a la población juvenil, en el campo de la educación no formal, en los sectores público, privado y mixto, desarrolladas en la ciudad de Medellín, Colombia, entre el 2000-2006. Se realizó con un enfoque hermenéutico aplicado al análisis de textos escritos, previo diseño de un sistema categorial y recolección de información, a través de una guía de revisión documental diseñada para tal fin, asimismo, el análisis de los datos se realizó manualmente. Se obtuvieron como resultado tres énfasis de formación en las propuestas: preventivo biológico, psico social y psico sociocultural.
\end{abstract}

PALABRAS CLAVE: JÓVENES * FORMACIÓN * SALUD * INTERVENCIÓN

ABSTRACT

This research has the purpose to carry out a state of the art of formative proposals in the field of health developed in the city of Medellin, Colombia, between 2000-2006, in the field of health, aiming at young population in the ambit of non-formal education. This research followed the hermeneutic approach applied to written text analysis, with a previous design of a categorical system and data collection, through a documental revision guide designed to such purpose. The data analysis was realized manually. We obtained as result three educational emphases in the proposals: preventive/biological, psycho-social and psycho-sociocultural.

KEYWORDS: YOUTH * TRAINING * HEALTH * INTERVENTION

Grupo de Investigación en Salud Mental de la Facultad Nacional de Salud Pública de la Universidad de Antioquia, Medellín, Colombia.

naeli46@yahoo.es 


\section{CONTEXTO}

El proyecto investigativo pretendió dar cuenta del estado del arte de las propuestas formativas con jóvenes en el campo de la educación no formal, área de salud, desarrolladas en Medellín, Colombia en los años 2000-2006.

Para la financiación de la propuesta, se contó con las instituciones que integran el nodo de conocimiento e información de la red de concertación de políticas públicas de juventud: la Escuela de Animación Juvenil, la Universidad Pontificia Bolivariana, la Corporación Región y la Subsecretaría de Metrojuventud de la Secretaría de Cultura Ciudadana de la Alcaldía de Medellín.

Como producto de la investigación se publicó el libro denominado: Formando juventudes: estado del arte de las propuestas formativas con jóvenes en el campo de la educación no formal en Medellín 2000 a 2006 (Londoño y García, 2008).
Se realizó una aproximación de las diferentes propuestas formativas implementadas con jóvenes en Medellín, con el fin de conocer los aspectos positivos alcanzados y conocimientos acumulados presentes en las instituciones/ organizaciones que brindan estos procesos de formación en la ciudad.

En estas condiciones se plantea el objetivo general siguiente: realizar un estado del arte de las propuestas formativas, orientadas a la población juvenil en el campo de la educación no formal, en los sectores público, privado y mixto, desarrolladas en la ciudad de Medellín entre el período 2000-2006.

En el presente artículo se presentarán los resultados en relación con el campo de formación para la salud. Las instituciones que hicieron parte de la muestra del estado del arte para el campo de formación salud, se relacionan en el cuadro 1.

\section{CUADRO 1 \\ PROPUESTAS FORMATIVAS CON JÓVENES, EN EL ÁMBITO DE SALUD \\ 2000- 2006}

\begin{tabular}{|c|c|c|}
\hline $\begin{array}{l}\text { CAMPO DE } \\
\text { FORMACIÓN }\end{array}$ & INSTITUCIÓN U ORGANIZACIÓN & NOMBRE DE LA PROPUESTA FORMATIVA \\
\hline \multirow{10}{*}{ Salud } & $\begin{array}{l}\text { Asociación por el bienestar de la } \\
\text { familia colombiana (Profamilia). }\end{array}$ & Jóvenes multiplicadores(as) (Profamilia, 2003). \\
\hline & $\begin{array}{l}\text { Centro de recursos integrales para la } \\
\text { familia (CERFAMI). }\end{array}$ & $\begin{array}{l}\text { Abogadores juveniles, por la salud y los derechos sexuales y } \\
\text { reproductivos de los(as) jóvenes. }\end{array}$ \\
\hline & & $\begin{array}{l}\text { Prevención de la violencia contra las mujeres, en las comunas } \\
1 \text { a } 4 \text { (CERFAMI, 2004). }\end{array}$ \\
\hline & $\begin{array}{l}\text { Caja de compensación familiar de } \\
\text { Antioquia (Comfama). }\end{array}$ & Gestores en salud sexual y reproductiva (Comfama, 2005). \\
\hline & Corporación Ser Humano. & $\begin{array}{l}\text { Círculos de promoción de la salud (Gómez, Bedoya y } \\
\text { Granda, 2003). }\end{array}$ \\
\hline & $\begin{array}{l}\text { Universidad de Antioquia, Ser Humano } \\
\text { y Surgir. }\end{array}$ & $\begin{array}{l}\text { Red de jóvenes para la prevención de la sexualidad insegura, } \\
\text { la farmacodependencia y la violencia, etapa de } 0 \text { a } 2 \\
\text { (Secretaría de Salud, 2006a). }\end{array}$ \\
\hline & Corporación Ser Especial. & $\begin{array}{l}\text { Necesidades educativas especiales y problemas } \\
\text { comportamentales de niños(as) y adolescentes (Sierra, 2007). }\end{array}$ \\
\hline & Fondo de Población Mundial. & $\begin{array}{l}\text { Proyecto Colombia (componente salud) (Proyecto Fondo } \\
\text { Mundial en Colombia, 2005). }\end{array}$ \\
\hline & $\begin{array}{l}\text { Oficina de la primera mujer y } \\
\text { Secretaría de las Mujeres de Medellín. }\end{array}$ & $\begin{array}{l}\text { No comer cuento, proyecto delgadez (Alcaldía de Medellín, } \\
2006 \text { ). }\end{array}$ \\
\hline & Corporación Surgir. & $\begin{array}{l}\text { Retomemos "Una propuesta para tomar en serio" (Surgir, } \\
2005 \text { ). }\end{array}$ \\
\hline
\end{tabular}


MÉTODOS

La investigación se realizó con un enfoque hermenéutico aplicado al análisis de textos escritos, se diseñó un sistema categorial (ver cuadro 2) y posteriormente, se realizó la recolección de información a través de una guía de revisión documental diseñada para tal fin. Asimismo, el análisis de los datos se realizó manualmente. El tipo de estudio fue exploratorio-descriptivo.
Cada una de las categorías se cruzó con algunas preguntas básicas que orientaron el norte del procesamiento de los datos, las preguntas seleccionadas fueron: ¿quiénes?, ¿con quiénes?, ¿dónde? y ¿cómo se ha hecho? La información brindada en los estados del arte permite clarificar cuáles son los desarrollos frente a un campo específico de indagación y en ese sentido, sugerir asuntos sobre el tema o situación de interés.

CUADRO 2

SISTEMA CATEGORIAL DE LA INVESTIGACIÓN FORMANDO JUVENTUDES

\begin{tabular}{|c|c|c|}
\hline $\begin{array}{l}\text { CATEGORÍAS } \\
\text { CENTRALES }\end{array}$ & DIMENSIÓN & INDICADORES \\
\hline $\begin{array}{l}\text { Instituciones u } \\
\text { organizaciones } \\
\text { que adelantan } \\
\text { los procesos } \\
\text { formativos. }\end{array}$ & $\begin{array}{l}\text { Identificación de la institución u } \\
\text { organización. } \\
\text { Misión y visión organizacional o } \\
\text { institucional. }\end{array}$ & $\begin{array}{l}\text { Nit (código de institución u organización). } \\
\text { Tipo (si es institución u organización). } \\
\text { Tipo de institución: oG, ong, caja de compensación, } \\
\text { universidad, alianza o red, otra. } \\
\text { Tipo de organización: grupos juveniles, formas de } \\
\text { articulación juvenil, organizaciones juveniles formales, otro. } \\
\text { Naturaleza de la institución: pública, privada, mixta, otra. } \\
\text { Dirección, teléfono, barrio de ubicación de la institución o de } \\
\text { la organización. } \\
\text { ¿La propuesta formativa explícita la misión y la visión? Sí/No }\end{array}$ \\
\hline $\begin{array}{l}\text { Contextos } \\
\text { que leen las } \\
\text { propuestas } \\
\text { formativas de los } \\
\text { y las jóvenes }\end{array}$ & $\begin{array}{l}\text { Diagnósticos realizados sobre la } \\
\text { juventud. } \\
\text { Escalas del contexto que leen: local, } \\
\text { nacional, global. } \\
\text { Problemas y potencialidades que } \\
\text { identifica en el contexto frente } \\
\text { a: situación de salud, de empleo, } \\
\text { educación y cultura, participación y } \\
\text { organización de los y las jóvenes. }\end{array}$ & $\begin{array}{l}\text { ¿La propuesta formativa parte de un diagnóstico? Sí/No } \\
\text { ¿La propuesta formativa lee el contexto de los(as) jóvenes? Sí/ } \\
\text { No } \\
\text { Variables y categorías del contexto en estos cuatro campos: } \\
\text { Formación para el trabajo, formación en salud, formación } \\
\text { ciudadana, formación artística. }\end{array}$ \\
\hline
\end{tabular}

\begin{tabular}{|c|c|c|}
\hline \multirow{9}{*}{$\begin{array}{l}\text { Sujetos } \\
\text { Se refiere a } \\
\text { quienes hacen } \\
\text { parte de los } \\
\text { procesos } \\
\text { formativos; } \\
\text { tanto los } \\
\text { sujetos jóvenes } \\
\text { en formación } \\
\text { como los sujetos } \\
\text { formadores. }\end{array}$} & \multirow{9}{*}{$\begin{array}{l}\text { Sujetos jóvenes en formación } \\
\text { Caracterización del sujeto joven } \\
\text { desde la propuesta: formativa. } \\
\text { ¿Quiénes son los jóvenes? }\end{array}$} & $\begin{array}{l}\text { Número de jóvenes formados(as) en las propuestas } \\
\text { formativas. }\end{array}$ \\
\hline & & Nivel socioeconómico de jóvenes formados(as). \\
\hline & & Lugares de procedencia. \\
\hline & & $\begin{array}{l}\text { Número de jóvenes con necesidades educativas especiales, } \\
\text { en situación de calle, en situación de desplazamiento, } \\
\text { pertenecientes a algún grupo étnico, unidad territorial a la } \\
\text { que pertenece, sector LGBT. }\end{array}$ \\
\hline & & Edades entre las que oscilan los(as) jóvenes. \\
\hline & & Nivel de escolaridad. \\
\hline & & $\begin{array}{l}\text { Número de hombres y mujeres jóvenes que participan en la } \\
\text { propuesta formativa. }\end{array}$ \\
\hline & & Expectativas, necesidades y demandas de formación. \\
\hline & & Concepción del sujeto joven: formas de nombrarlo y asumirlo. \\
\hline
\end{tabular}




\begin{tabular}{|c|c|c|}
\hline $\begin{array}{l}\text { CATEGORÍAS } \\
\text { CENTRALES }\end{array}$ & DIMENSIÓN & INDICADORES \\
\hline \multirow[t]{5}{*}{ Sujetos } & \multirow{5}{*}{$\begin{array}{l}\text { Sujetos formadores(as) } \\
\text { Tipificación de la formación de los } \\
\text { sujetos formadores. }\end{array}$} & $\begin{array}{l}\text { Edades entre las que oscilan los(as) formadores(as): } \\
\text { grupo etario al que pertenecen los(as) formadores(as). }\end{array}$ \\
\hline & & Nivel educativo de los(as) formadores(as). \\
\hline & & $\begin{array}{l}\text { Áreas de profesionalización: nro. de profesionales en } \\
\text { ciencias sociales, áreas de la salud u otros. }\end{array}$ \\
\hline & & Formación específica en juventud. \\
\hline & & Formas de nombrar y definir el sujeto formador. \\
\hline \multirow{2}{*}{$\begin{array}{l}\text { Intencionalidad } \\
\text { Es el para qué de la } \\
\text { acción formativa, lo que } \\
\text { se pretende lograr en el } \\
\text { sujeto en formación, en el } \\
\text { contexto y en su hacer. }\end{array}$} & $\begin{array}{l}\text { Objetivo(s), propósito(s) o fin(es) de } \\
\text { la formación. }\end{array}$ & $\begin{array}{l}\text { Se explicita: } \\
\text { El objetivo Sí/No } \\
\text { El propósito Sí/No } \\
\text { Los fines Sí/No. }\end{array}$ \\
\hline & $\begin{array}{l}\text { Concepción de sujeto joven que quiere } \\
\text { formar. }\end{array}$ & Se explicita qué tipo de sujeto joven se quiere formar. \\
\hline
\end{tabular}

\section{Enfoque de formación de Concepción de formación.}

jóvenes y conceptual desde donde se realiza el proceso formativo.

Concepción de educación no formal.

Denominación de la práctica ¿Explicita la denominación de su práctica educativa? educativa. $\quad$ Sí/No

Referentes teóricos y conceptuales ¿Explicitan desde qué referentes teóricos y que orientan la propuesta formativa. conceptuales orientan su propuesta formativa? Sí/No

Reconocimiento de las características ¿Explicita el reconocimiento de la diversidad juvenil? y situaciones de la población juvenil (estrato socioeconómico, género, etnia, necesidades educativas especiales, rural-urbana y diversidad sexual).

Coherencia entre los componentes de la propuesta formativa: lectura del contexto, sujetos, intencionalidades, enfoque de formación, metodologías y contenidos.

$\begin{array}{ll}\text { Incidencia del sujeto joven en } & \text { ¿La propuesta formativa explicita las formas de } \\ \text { formación en la construcción y } & \text { participación de los jóvenes? Sí/No }\end{array}$ formación en la construcción y participación de los jóvenes? Sí/No recreación de la propuesta formativa.

\section{Metodología}

Es la coherencia entre objetivos, instrumentos y prácticas; es decir, la coherencia global del proceso.

\section{Criterios metodológicos. \\ ¿La propuesta explicita los criterios metodológicos?}

Estrategias metodológicas (modelos ¿La propuesta explicita estrategias metodológicas y o patrones del procedimiento metodológico).

Diseños metodológicos (actividades $\mathrm{y}$ rutas). diseños metodológicos? Sí/No

\section{CATEGORÍAS \\ CENTRALES}

\section{DIMENSIÓN}

Nivel de formalización de la propuesta curricular: Plan o programa de contenidos, criterios $\mathrm{y}$ orientaciones generales de los contenidos.

\section{INDICADORES}

Intensidad horaria.

Es el qué de la propuesta formativa; pueden estar formalizados en un plan de estudios, programa o propuesta curricular o bien, pueden existir unos criterios y orientaciones generales. 
CRITERIOS DE INCLUSIÓN EN LA MUESTRA DOCUMENTAL

a) Instituciones u organizaciones que tengan propuestas formativas entre los años 2000 y el 2006, ejecutadas en Medellín o sus corregimientos.

b) La propuesta de formación no necesariamente debe estar reportada en una base de datos especializada.

c) Accesibilidad a la información escrita.

d) Propuestas formalizadas en un plan de estudios, programa o currículo.

e) Instituciones u organizaciones que tuvieran interés en hacer parte del estudio.

f) No se consideran las propuestas formativas del ámbito de la educación formal o las que tengan como propósito que los $y$ las jóvenes ingresen a la educación básica primaria o secundaria.

g) Se incluyeron instituciones de rehabilitación, solo si tenían trabajo comunitario, es decir, extramural; asimismo, que cumplieran con los criterios mencionados anteriormente.

En la ciudad de Medellín, se identificaron catorce propuestas de formación en salud, realizadas con jóvenes en el ámbito no formal, durante el tiempo considerado por el estudio. De estas catorce que ingresaron a la base de datos (los resultados cuantitativos no se presentan en el presente artículo) se sometió a un proceso de análisis hermenéutico a nueve propuestas de formación, las cuales cumplían con los criterios de selección de la muestra. Las cinco que no ingresaron al estudio no cumplieron con los criterios de accesibilidad y(o) la propuesta formativa no estaba materializada en un texto, tal como plan de estudio, programa o currículo.

\section{RESULTADOS}

CONTEXTOS PARA LA INTERVENCIÓN EN LAS PROPUESTAS FORMATIVAS EN SALUD

La lectura del contexto se centra en los problemas de los(as) jóvenes, básicamente en estadísticas y estudios epidemiológicos, como prevalencias e incidencias de los embarazos precoces, adquisición de infecciones de transmisión sexual (ITS), uso de sustancias psicoactivas, suicidios y actos violentos. Además, se observa que los estudios retomados para el contexto no son locales, tienen un carácter nacional o internacional.

Los estudios de situación de salud de la población juvenil, señalan las drogodependencias, el consumo indebido de sustancias psicoactivas, los riesgos derivados del ejercicio de una sexualidad insegura y la violencia, como los tres principales problemas de salud, que afectan este grupo poblacional (Agudelo, Montoya, Gallo, Lopera, López, Saldarriaga y Vásquez, 2005: 15).

Los temas abordados son los que intervienen en las propuestas de formación, las cuales dejan de lado una delimitación del contexto en cuanto a las fortalezas de los(as) jóvenes y dedican pocos párrafos para resaltar la resiliencia juvenil o los indicadores de bienestar de la población de naturaleza cuantitativos y cualitativos.

En cuanto a la realización de diagnósticos, las propuestas de formación como las de Profamilia, el Fondo Mundial, la Red de Jóvenes, Comfama, Surgir y Proyecto Delgadez, no parten de estudios específicos (líneas de base) sobre la población a la cual van dirigidas sus acciones, sino que retoman estudios nacionales y mundiales que les sirven de sustento para justificar sus acciones $y$ diseñar sus estrategias.

La Corporación Ser Humano lleva a cabo una especie de metodología denominada "diagnosticar-haciendo", lo cual ayuda a que se pueda hacer no solo un seguimiento de la evolución en los cambios de percepción de los jóvenes frente a la salud y a la enfermedad, sino también establecer niveles comprensivos en los chicos y las chicas, en torno a las problemáticas $y$ sus posibles soluciones.

Por su parte, la Corporación Ser Especial solo realiza diagnósticos uno a uno, derivados del encuentro entre psicoanalista y niño(a) o joven. No hacen uso de técnicas evaluativas desprendidas de la estadística ni de 
la epidemiología; considera que cada sujeto es único, por lo tanto, no es representable en una estadística. En esta vía, las estrategias de trabajo con los chicos y chicas son personalizadas, al igual que el trabajo con las familias.

\section{SUJETOS QUE SE EVIDENCIAN EN LA PROPUESTA FORMATIVA}

A partir de una lectura de los contextos y diagnósticos basados para la mayoría de propuestas formativas en una mirada desde la epidemiología clásica y la salud pública, la cual asume factores de riesgo y protección, la concepción de sujeto que más se presenta en las propuestas formativas parte de la noción de "vulnerable".

La mayoría de las propuestas formativas han acuñado los conceptos vulnerable $y$ vulnerabilidad, para hablar de la población juvenil que está expuesta a focos de enfermedad. El concepto vulnerable se ha trasladado de las probabilidades estadísticas, a una forma de nombrar las características sociales, económicas y políticas de un sujeto o una población.

En el análisis de los datos del presente estado del arte fue difícil rastrear la diferencia entre cuál es la concepción de sujeto joven propio de la propuesta formativa y cuál es el sujeto joven que se quiere formar. La primera habla del joven que entra a formarse en la propuesta $y$ la segunda hace referencia del que se desea formar; uno real $y$ uno ideal, cuya diferencia no siempre es visible fácilmente en los textos analizados.

Se observó que para una de las propuestas de formación correspondiente a la corporación Ser Especial, se tiene una construcción de sujeto desde el inconsciente, en la cual lo cronológico y lo orgánico no ocupan el primer lugar. Es un sujeto del lenguaje (lo social, lo político y lo cultural son creaciones del lenguaje); es decir, "sujeto" a su propia construcción de signos y significados que lo convierten en un ser que padece, demanda, goza y vive; ese mismo vínculo con el lenguaje es lo que lo puede sacar de sus padecimientos. Durante el análisis realizado en los textos, no se pudo rastrear la noción de sujeto formador, las propuestas no cuentan con acápites explícitos para ello.

\section{INTENCIONALIDAD DE LAS PROPUESTAS FORMATIVAS}

Cada propuesta formativa tiene fines específicos. Algunas están centradas en la promoción de la salud, otras en la prevención de la enfermedad, otras se enfocan en el género $y$ otras buscan espacios de reconocimiento de la subjetividad de aquellos(as) jóvenes que tienen algún tipo de trastorno mental y del comportamiento: "una intencionalidad: que la vivencia del cuerpo fuese posible partir del goce, el disfrute, la alegría, la sensualidad, el contacto y en general, las sensaciones placenteras" (Proyecto Fondo Mundial en Colombia, 2005a: 13).

La Corporación Ser Humano y el Proyecto Delgadez, teóricamente se basan en el concepto de promoción de la salud para la intervención, enfatizan en que esta no se puede entender quitándola de sus marcos políticos $y$ culturales. Resaltan el peligro de poner la responsabilidad de la salud solo en los estilos de vida saludables, en los cuales son los sujetos los únicos responsables de los deterioros de su salud, desconociendo las responsabilidades de los gobiernos, que son los que realimentan macro-estructuras sociales donde están inmersos los(as) jóvenes que promueven la enfermedad $y$ no la salud. Por otro lado, explican que los determinantes macro estructurales son asuntos clave para comprender $y$ atacar los problemas de salud de una población.

En particular, la Corporación Ser Humano centra su atención en los determinantes culturales de la salud y la enfermedad, rescata la posibilidad que tienen los seres humanos de trasformar su entorno cultural para que finalmente, este se revierta en la salud de los sujetos.

La Corporación Ser Especial considera que el psicoanálisis es la vía para trabajar los desórdenes de los(as) adolescentes que atiende. Además, dentro de sus propósitos se encuentra posicionar el psicoanálisis en la esfera pública, como terapia, como abordaje de la psique $y$ como área de conocimiento, que desea incidir por medio de su lectura psicoanalítica en los síntomas contemporáneos de los(as) adolescentes - esta última es la manera como denominan al sujeto joven. 
CARACTERÍSTICAS Y SITUACIONES ESPECIALES DE LA POBLACIÓN JUVENIL

Fue difícil rastrear la inclusión de la diversidad juvenil en las propuestas formativas de acuerdo a las categorías de estrato socioeconómico, jóvenes rurales o urbanos, etnia $y$ diversidad sexual. Sin embargo, para el campo de la salud es evidente que el grupo poblacional al cual van dirigidas las acciones en salud sexual y reproductiva, así como en las dirigidas a la anorexibulimia, corresponden en mayor proporción a las mujeres. Con relación a los jóvenes varones, las propuestas de formación están centradas en la prevención de la farmacodependencia.

Dentro de las propuestas formativas en salud que hacen énfasis en las mujeres, es poco frecuente encontrar estrategias que trabajen tipos de identidades femeninas, analíticas y empoderadas, así como que promuevan resistencias a las propuestas de formación humana centradas en estereotipos dañinos sobre lo femenino. La propuesta de prevención de la anorexibulimia es la que más énfasis realiza sobre la construcción de la identidad femenina. La salud y el cuerpo de las mujeres no se reconocen como un posible campo político de estudio; al contrario, prima el enfoque de prevención de la enfermedad, de desórdenes orgánicos, embarazos o infecciones de transmisión sexual.

\section{TIEMPOS DE LA FORMACIÓN}

En los contenidos, se puede observar que en su mayoría siempre son muy amplios, en contraposición con los tiempos cortos en que se da la formación, lo que dificulta la problematización, conceptualización y la reflexión sobre las posturas y prácticas que asumen $\operatorname{los}(\mathrm{as})$ jóvenes frente a los comportamientos de riesgo o protectores. Los tiempos de formación están estructurados en correlación con los objetivos de la propuesta, $y$ estos varían entre horas $y$ semanas, tal y como se observa en la siguiente propuesta formativa: Para la multiplicación, lea con atención el módulo 7 y según vaya a un grupo del sector salud, familia, educación, facilite los 3 primeros módulos completos, más el abanico que corresponde del 4, para un total de 12 sesiones o talleres educativos (Alcaldía de Medellín, 2006: 1).

\section{COOPERANTES}

A excepción de las corporaciones Ser Especial y Ser Humano, de quienes no se tiene información sobre recepción de cooperación extranjera, las demás instituciones reciben dinero de cooperantes extranjeros para llevar a cabo sus propuestas formativas. Los enfoques de formación obviamente son tocados por los supuestos teóricos o metodológicos de los que parte el cooperante internacional. La mayoría de ellos se enfocan en la prevención y detección temprana de la violencia, suicidio, embarazo temprano $y$ adquisición de ITs, con un énfasis en la reducción del daño e intervención en factores de riesgo para la salud.

\section{SISTEMAS DE EVALUACIÓN}

Los sistemas de evaluación y monitoreo de las propuestas formativas aún son precarios y en su mayoría, no son reportados en los documentos analizados. Las propuestas que reportan algún tipo de evaluación (Comfama, Universidad de Antioquia y Proyecto Colombia) realizan las evaluaciones sobre las actividades, cobertura y cumplimiento, pero no se conocen los resultados cualitativos ni el impacto sobre los(as) jóvenes que participan del programa.

\section{ENFOQUES METODOLÓGICOS DE LAS PROPUESTAS FORMATIVAS}

Como es de esperarse, la elección de los enfoques afecta las metodologías utilizadas en la propuesta formativa y probablemente, la vida de los(as) jóvenes participantes, sobre todo en el modo de configurar y concebir la salud, la enfermedad y el cuerpo. Las propuestas de formación que centran sus acciones en enfoques biológicos y comportamentales (positivistas) ponen el énfasis en los síntomas corporales y en los problemas de comportamiento de los(as) jóvenes. Aquellas que se basan en enfoques psicológicos y socio-antropológicos (comprensivos) focalizan los síntomas 
psicológicos y su expresión en las responsabilidades subjetivas y sociales de los problemas de salud que aquejan a los(as) jóvenes.

En los textos analizados se observa que las dos estrategias de intervención más utilizadas desde el punto de vista de la formación, son la promoción de la salud y la prevención de la enfermedad. Cuando las propuestas de formación se centran en la promoción de la salud, se ubican en la promoción de un sujeto socio-crítico, políticamente dialógico, con capacidad de interpelar sus propias prácticas y las comunitarias, además, construye conciencia crítica sobre los sistemas económicos $y$ políticos en torno a la salud y el cuerpo de los(as) jóvenes, haciendo énfasis en los usos personales y sociales del cuerpo.

Las estrategias que se ubican en el lado de la prevención de la enfermedad, desean atenuar factores de riesgo personales y comunitarios, como prevenir la adquisición de una ITS o el consumo de sustancias psicoactivas mediante "prevención primaria"; reducir la posibilidad de un contagio de una ITS o aminorar sus secuelas y prevenir el uso indebido de sustancias psicoactivas mediante "prevención secundaria" y por último, aliviar las secuelas de la ITS $y$ de la drogodependencia para evitar el agravamiento de las enfermedades por medio de "prevención terciaria".

Ambas estrategias (de promoción y prevención) tienen un eje central que es la intervención sobre el cuerpo de los(as) jóvenes, con el fin de incidir sobre el plano físico, en el "cuerpo" o en la representación del mismo, la "corporalidad"; a la vez que desean analizar y modificar las prácticas sobre este:
Éste modulo desarrolla la propuesta pedagógica de la corporación Ser Humano, construida a lo largo de siete años con base en una experiencia pedagógica-práctica, afianzada conceptualmente en los enfoques psicosocial y socio-cultural, inscritos en el paradigma humanista de la promoción de la salud (Gómez, Bedoya y Granda, 2003: 18)

En cuanto a los dispositivos de las metodologías, se habla de taller y de otras propuestas interactivas como video juegos $y$ robots que asemejan bebes para el tema de embarazo adolescente, así como, las artes plásticas como técnica y dispositivo de aprendizaje.

\section{CONCLUSIONES}

Las propuestas formativas construyen sus énfasis retomando aspectos históricos de la epistemología del área de la salud, definida a partir de dos enfoques predominantes $y$ tres escuelas de pensamiento: a) el biológico, comportamental, médico y tradicional (enfoque positivista); b) los enfoques psicológicos, antropológicos y de la medicina (los más cercanos a los enfoques comprensivos) y c) la epidemiología social o socio-crítica, (la línea más cercana a retomar los enfoques comprensivos). Cada enfoque ha tratado de poner en relieve diferentes nociones de lo que es la salud, la enfermedad, el cuerpo y como fin último, la vida y la muerte. Derivado de estos dos enfoques $y$ tres escuelas de pensamiento, en el estado del arte se encuentran tres énfasis en la formación en salud. 


\author{
GRÁFICO 1 \\ ÉNFASIS EN LA FORMACIÓN EN EL CAMPO DE LA SALUD \\ EMERGENTES EN EL ESTADO DEL ARTE \\ 2000-2006
}

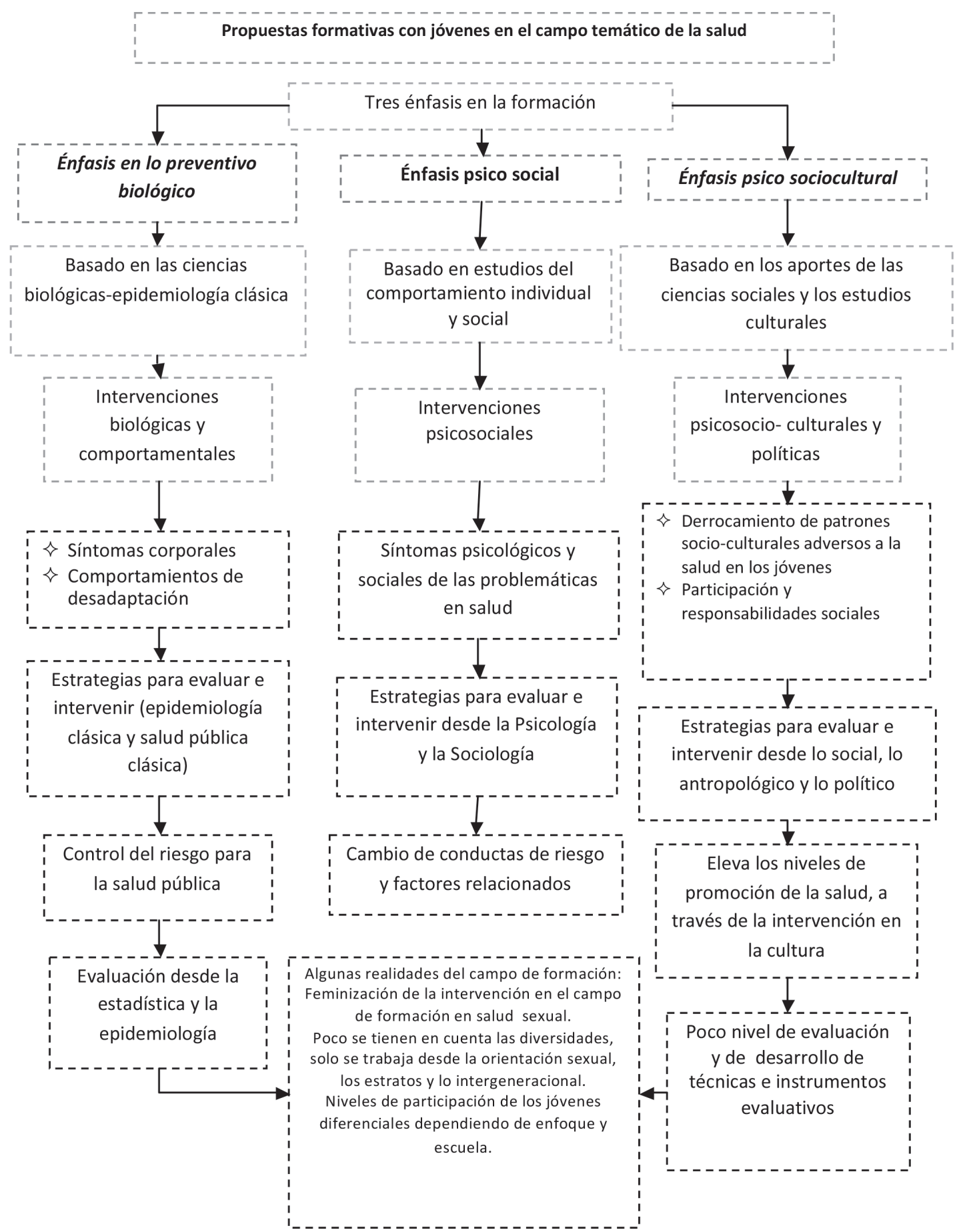


Otras conclusiones giran en torno a los siguientes asuntos:

El sujeto formador y su lugar en las propuestas formativas, no aparecen explícitamente en los documentos analizados. Asimismo, fue difícil rastrear la inclusión de la diversidad juvenil de acuerdo a las categorías de estrato socioeconómico, jóvenes rurales o urbanos, etnia $y$ diversidad sexual.

Por otro lado, se observa la existencia de algunos temas enfocados exclusivamente para los hombres y otros temas que se relacionan con mujeres. En este caso, la formación en salud sexual y reproductiva es predominante para las mujeres $y$ lo referente a la prevención en farmacodependencia para los hombres.

En cuanto a los contenidos, se observó que casi siempre son muy amplios; no obstante, los tiempos de formación son breves, lo cual genera dificultades en la comprensión y reflexión de los jóvenes ante situaciones de riesgo.

Existe diversidad de enfoques, trabajo interdisciplinario e interés por la construcción de estrategias de formación, mostrando avances en la superación de la existencia única de la mirada a la salud desde lo biologicista-positivista, acogiendo cada vez más paradigmas socio-críticos $y$ hermenéuticos.

Se observa un interés incipiente por construir nichos teóricos para entender los procesos de salud y enfermedad de las comunidades $y$ los grupos poblacionales, desde una perspectiva multidisciplinaria que articula teorías de los determinantes de salud acuñados por corrientes de la salud pública, la epidemiología, la psicología social, la antropología, etc., articulándolos a una lectura de los contextos de los sujetos, los estudios ecológicos, su ambiente y el medio. No aparece en los textos analizados la posibilidad de discusión de los diferentes énfasis en salud $y$ que compartan metodologías exitosas entre las instituciones formadoras.

Otro aspecto que no se evidencia en los textos analizados es la oportunidad de discusión de los diferentes énfasis en salud, así como, la posibilidad de vincular las metodologías propuestas entre las instituciones formadoras.
Algunas de las propuestas de formación analizadas, están ancladas a políticas institucionales. Sin embargo, el tema de la salud en la juventud, es la razón de ser institucional y por ende, no está sujeta a la oscilación de voluntades políticas. Aún así, durante los años seleccionados para el estudio, se observó que la cooperación internacional jugó un papel importante en el traslado de recursos para las propuestas de formación con jóvenes en el área de la salud. Finalmente, no aparecen evaluaciones de impacto de los programas.

\section{DISCUSIÓN}

La existencia de acuerdos, plataformas, convenios y reglamentación nacionales e internacionales en el ámbito de la salud juvenil, brindan parámetros para el análisis en materia de salud pública y co-morbilidades asociadas a los problemas de salud de los(as) jóvenes; asimismo sostiene la necesidad de reglamentar, normatizar y aportar a la construcción de pautas de actuación. Dichos acuerdos han puesto en las agendas locales, la temática de la salud en los mundos juveniles.

Sin embargo, según los documentos analizados en el estado del arte, aún persisten dificultades en la implementación de estudios cuantitativos y epidemiológicos de la situación de salud de los(as) jóvenes, para conseguir perfiles de morbi-mortalidad juvenil más exactos. Además, existen impedimentos para construir modelos de investigación que permitan asociar los perfiles epidemiológicos al modelo(s) de desarrollo del país y a las dinámicas del contexto social y político en el que se desarrolla la juventud en ciudades en vía de desarrollo, como Medellín, Colombia.

Se está de acuerdo con la investigación de Hernán, Ramos y Fernández (2001), quienes plantean que las intervenciones en el campo de la salud con jóvenes, no son evaluadas continua e integralmente con el propósito de conocer el impacto y el programa de la intervención.

Pese a que investigaciones realizadas en la ciudad demuestran una relación directa entre enfermedad $y$ condiciones político-sociales $y$ militares (Agudelo, Montoya, Gallo, Lopera, López, Saldarriaga y Vásquez, 2005; Agudelo, 
Gómez y López, 2007), las propuestas retoman escasamente esta relación para la construcción de los currículos de formación.

A pesar de que en la ciudad y en el continente se han realizado algunos estudios sobre la asociación de la cultura con los problemas de la sexualidad juvenil (De la Cuesta, 2005 y Boceara, 2007) aun los currículos de capacitación $y$ las acciones de prevención no hacen uso de dichos hallazgos para construir currículos que logren mayor efectividad en la intervención.

Las investigaciones realizadas en Medellín, han enfatizado en que los currículos de capacitación solo llegan hasta el ámbito de la adquisición de conocimientos, pero no cambian las creencias y las actitudes de los jóvenes, las cuales son las bases para las enfermedades que aparecen en sus vidas, sobre todo las relacionadas con lo psicosocial (Gallo, 2008).

De igual manera, no se proponen currículos de intervención donde se dé un lugar protagónico a los encuentros inter-generacionales, factor determinante para el éxito de las intervenciones en salud en el ámbito juvenil (Gallo, 2009).

\section{BIBLIOGRAFÍA}

\section{LIBROS}

Agudelo, L. M.; Montoya, E. M.; Gallo, N. E.; Lopera, G. E; López, L. M.; Saldarriaga, L. M. y Vásquez, C. E. Redescubrir una mirada a la salud de los jóvenes. Prevalencias en embarazos adolescentes, sintoma sugestivo de ITS, consumo $y$ adicción a sustancias psicoactivas, comportamientos violentos y factores relacionados en jóvenes de 14 a 19 años. Módulo 1. Medellín: Alcaldía de MedellínEditorial Multimpresos LTDA, 2005.

De la Cuesta, C. Tomarse el amor en serio. Contexto del embarazo adolescente en la ciudad de Medellín. Medellín: Universidad de Antioquia, 2005.

Gómez, A.; Bedoya, M. y Granda, T. "Concepciones sobre promoción de la salud y prevención de la enfermedad". Serie: la salud en los mundos juveniles. Módulos 1-3. Medellín: Corporación Ser Humano-Alcaldía de Medellín-Secretaría de Salud-Plan de Prevención a la Drogadicción, 2003.

Londoño, J.; Gallo, N. E. y García, S. Formando juventudes: estado del arte de las propuestas formativas con jóvenes en el campo de la educación no formal en Medellín 2000 a 2006. Medellín: Corporación Región, 2008.

Proyecto Fondo Mundial en Colombia. "Instrucciones, convenciones y coordenada". Construcción de una respuesta intersectorial en salud sexual y reproductiva, con énfasis en prevención y atención a las ITS-VIH-SIDA, con jóvenes y adolescentes residentes en comunidades receptoras de población desplazada en Colombia. Módulo 1. Bogotá: Taller de Edición, 2005a.

Proyecto Fondo Mundial en Colombia. "Educación para la sexualidad". Construcción de una respuesta intersectorial en salud sexual y reproductiva, con énfasis en prevención $y$ atención a las ITS-VIH-SIDA, con jóvenes y adolescentes residentes en comunidades receptoras de población desplazada en Colombia. Módulo 3. Bogotá: Taller de Edición, 2005b.

Proyecto Fondo Mundial en Colombia. "Organización y emprendimientos juveniles". Construcción de una respuesta intersectorial en salud sexual y reproductiva, con énfasis en prevención y atención a las ITS-VIH-SIDA, con jóvenes y adolescentes residentes en comunidades receptoras de población desplazada en Colombia. Módulo 6. Bogotá: Taller de Edición, 2005c.

Proyecto Fondo Mundial en Colombia. "Memoria de una permanente expedición". Construcción de una respuesta intersectorial en salud sexual y reproductiva, con énfasis en prevención y atención a las ITS-VIH-SIDA, con jóvenes y adolescentes residentes en comunidades receptoras de población desplazada en Colombia. Módulo 8. Bogotá: Taller de Edición, 2005d.

Secretaría de Salud de Medellín. "Proyecto Red de jóvenes para la prevención de 
la farmacodependencia, la sexualidad insegura y la violencia”. Etapa II. Redescubrirte: enhebrando saberes en salud y juventud. Módulo 1. Medellín: La Secretaría, 2006a.

Secretaría de Salud de Medellín. "Proyecto Red de jóvenes para la prevención de la farmacodependencia, la sexualidad insegura y la violencia”. Etapa II. Redescubrirte: enhebrando saberes en salud y juventud. Módulo 2. Medellín: La Secretaría, 2006b.

Secretaría de Salud de Medellín. "Lineamientos para la formulación de proyectos. Proyecto Red de jóvenes para la prevención de la farmacodependencia, la sexualidad insegura y la violencia”. Etapa II. Redescubrirte: enhebrando saberes en salud y juventud. Módulo 3. Medellín: La Secretaría, $2006 c$.

Secretaría de Salud de Medellín. "Una propuesta para el trabajo con jóvenes. Proyecto Red de jóvenes para la prevención de la farmacodependencia, la sexualidad insegura y la violencia”. Etapa II. Redescubrirte: enhebrando saberes en salud y juventud. Módulo 4. Medellín: La Secretaría, 2006d.

Sierra, G. ¿Sin límite? el extravío de la autoridad $y$ su efecto en niños $y$ adolescentes. Medellín: Corporación Ser Especial, 2007.

\section{REVISTAS}

Agudelo, L.M.; Gómez, J.A. y López, A. "Representaciones sociales: otra perspectiva de estudio de la violencia". Revista Colombiana de Psiquiatría 36 (2). 2007: 1-14. En: <http://scielo. isciii.es/scielo.php?script $=\mathrm{sci}$ artte $\mathrm{xt} \& \mathrm{pid}=\mathrm{S} 1135-57272001000600002$ \&lng $=$ es\&nrm $=$ iso $>$.

Boceara, G.B. "Etnogubernamentalidad: la formación del campo de la salud intercultural en chile. Chungará (Arica)". Revista de Antropología Chilena 39 (2). 2007:185-207. En: <http://www. scielo.cl/pdf/chungara/v39n2/art03.pdf $>$ [consultado el 21 de mayo de 2012].
Gallo, N.E. y Molina A. N. "Evaluación de resultados inmediatos del proyecto acciones de información, educación, y comunicación en salud sexual y reproductiva para Medellín en 2007". Revista Salud Pública de Medellín 3 (1). 2008:47-62. En: <http:// www.medellin.gov.co/irj/go/km/docs/ wpccontent/Sites/Subportal $\% 20 \mathrm{del} \% 20$ Ciudadano/Salud/Secciones/Plantillas\%20 Gen\%C3\%A9ricas/Documentos/2012/ Revista $\% 20 \mathrm{Salud} / \mathrm{Rev}$ is ta $\% 20$ Vol.\%203\%20N\%C2\%B0\%201/4.\%20 Evaluaci\%C3\%B3n\%20de\%20resultados. pdf $>$.

Gallo, N. E. "Línea de base del proyecto salud sexual y reproductiva para adolescentes en Medellín: componente cualitativo". Rev. Fac. Nac. Salud Pública 27 (3). 2009: 282-290. En: <www.redalyc.org/ articulo.oa?id=12016344005>.

Hernán, M.; Ramos, M. y Fernández, A. "Revisión de los trabajos publicados sobre promoción de la salud en jóvenes españoles". Rev. Esp. Salud Pública 75 (6). 2001: 491-504. En: <www.msssi.gob. es/biblioPublic/publicaciones/recursos propios/resp/ revista cdrom/vol75/ vol75 6/RS756C 491.pdf $>$.

TESIS

Trilla, J. "Educación fuera de la escuela, la educación a distancia, por correo, internet, radio, video y otros medios de comunicación no formales". Barcelona: Planeta, 2005.

Manco, D.; Puerta, J. y Taborda, P. "Procesos educativos alternativos que potencian la formación de sujetos sociales". [Tesis]. Medellín: Universidad de Antioquia; 2006.

\section{TEXTOS ELECTRÓNICOS}

Alcaldía de Medellín. No comer cuento. Guía de procesos para formación y multiplicación, campaña de prevención de la anorexi-bulimia en mujeres en la ciudad de Medellín. Medellín: Secretaría de Salud, 2006. En: <http://www. 
medellincultura.gov.co/medellinjoven/ Documents/2008_observatorio_ juventud/Formando\%20Juventudes.pdf $>$ [consultado el 10 de junio de 2012].

Programa de Cooperación Sur-Sur en Salud Sexual y Reproductiva. Salud sexual y reproductiva para adolescentes en América Latina. Aplicando las lecciones aprendidas desde la cooperación sursur. 2006. En:<http://www.profamilia. com/images/stories/ afiches/libros/libros/ corporacion-sur-sur.pdf $>$ [consultado el 25 de junio de 2006].

SURGIR. Retomemos: una propuesta para tomar en serio. Medellín: Corporación SURGIR, 2005. En: <www.corporacionsurgir.org/ Publicaciones/Cr\%f3nica\%20de\%20 un $\% 20$ compromiso.pdf $>$ [consultado el 10 de junio de 2012].
OTROS

Caja de Compensación Familiar de Antioquia (CомғамA). Gestores en salud sexual y reproductiva. 2005.

Centro de Recursos Integrales para la Familia (CERFAMI). Abogadores juveniles por la salud y los derechos sexuales $y$ reproductivos. CERFAMI, 2004.

Centro de Recursos Integrales para la Familia (CERfAMI). Informe Proyecto Prevención de la Violencia de Género en Mujeres Adolescentes de las Comunas 1 a la 4 en la Ciudad de Medellín. Cerfami, 2006.

Fecha de ingreso: 05/07/2013 Fecha de aprobación: 29/11/2013 
